Die Rückständigkeit unserer offiziellen Medizinalstatistik hat wohl niemand stärker empfunden als $\mathrm{Pr}$ in $z$ ing selbst, der in seinen zahlreichen Arbeiten und vor allem in seinem weltbekannten „Handbuch der medizinischen Statistik" die statistischen Materialien aus verschiedenen Ländern zusammenstellte und verarbeitete und hierbei in vielen Fällen auf deutsche Vergleichszahlen verzichten mußte. Wohl jeder, der die Arbeiten P ri n z ing s kennt, wird gestehen, daß die medizinische Statistik nicht die spröde Materie darstellt, für welche sie vielfach noch heute gehalten wird, denn es kommt hierbei vor allem darauf an, von wem sie dargestellt wird. Ich selbst muß gestehen, daß das Studium der Schriften $\mathrm{Pr}$ in $z$ ing s für mich von bestimmendem Einfluß war. Der großell sozialen und soziologischen Bedeutung der Medizin und ihrer Statistik wurde ich erst nach vollendetem Universitätsstudium durch die Lektüre dieser Schriften bewußt in denen zahlreiche medizinische und hygienische Probleme aufgerollt und mit klarem und kritischem Blick vom statistischen Standpunkt aus behandelt sind. Was die Arbeiten Prinz ing s vor allem auszeichnet, ist ihre statistische Zuverlässigkeit und Gründlichkeit, ihre strenge Objektivität und kritische Beweisführung. Nur wer sich selbst schon mit statistischen Arbeiten befaßt hat, wird die von $\mathrm{Pr}$ in $\mathrm{z}$ in $\mathrm{g}$ geleistete Arbeit richtig zu würdigen verstehen. Dagegen dürfte es manchem unverständlich sein, wie ein Privatstatistiker, ein Privatarzt, ohne die Hilfsmittel eines statistischen Bureaus eine solche ArbeitsIeistung überhaupt $\mathrm{zu}$ verrichten vermochte. In Anbetracht solcher Verdienste um einen in Deutschland fast völlig brachliegenden, jedoch hoffnungsvollen $Z$ weig der medizinischen Wissenschaft ist es begreifiich, da $\beta$ das Gefühl der Dankesschuld für seine uneigennützige und von einer idealen Hingabe beseelte Arbeitsleistung von allen Aerzten empfunden wird, die wissenschaftlichen oder praktischen Nutzen aus seinen Schriften gezogen haben. Diese Aerzte verteilen sich über die ganze Welt, denn man findet seinen Namen in der Fachliteratur selbst der entlegensten Länder zitiert. Es ist bekannt, daß man in der russischen Fachliteratur seinem Namen sogar noch häufiger begegnet als in der deutschen.

Glücklicherweise ist es statistisch nachgewiesen, daß schon viele Statistiker sehr alt geworden sind und selbst im Alter von über 80 Jahren noch sehr produktiv waren. Auch $\mathrm{P}$ r i n $z$ in $g$ scheint nicht aus der Art zu schlagen; denn wir werden von ihm als Jubiliäumsspende noch in diesem Jahre die zweite Ausgabe des ersten Teiles seines Handbuches der medizinischen Statistik erhalten. So ehrt und beschenkt ein rastlos arbeitender Meister seine Schüler.

$$
\text { E. Roes le (Berlin). }
$$

\section{Zum 70. Geburtstage von Friedrich Prinzing.}

Am 3. IV. 1929 feierte F r i e d r i c h Prin z in g in seiner Heimatstadt Ulm a. D. seinen 70 . Geburtstag. Nur wenige hatten infolge der großen Bescheidenheit dieses Mannes davon auf Umwegen Kenntnis erhalten; deshalb wurde auch die Schriftleitung dieser Zeitschrift erst post festum darauf aufmerksam gemacht und können die großen Verdienste ihres langjährigen Mitarbeiters um die Entwicklung eilles Sondergebietes der medizinischen Wissenschaft erst heute hier gewürdigt werden.

Prinz ing bildet das seltene Beispiel dafür, daß ein praktischer Arzt in einer Provinzstadt ein Meister auf einem Gebiete wird, das zu der Tätigkeit des praktischen Arztes überhaupt nicht gehört und wegen seiner schwierigen Methodik und aus naheliegenden anderen Gründen von den Aerzten, und zwar nicht allein von den praktischen, im allgemeinen ängstlich gemieden wird, nämlich auf dem Gebiet der medizinischen Statistik. Solche Verdienste wurden allerdings vom State viel weniger gewürdigt, als z. B. in England, wo man schon zu den Zeiten-W ill i a $\mathrm{m} \mathrm{F}$ a r r s die Seltenheit der statistischen Begabung eines Arztes für die offizielle Statistik des Staates auszunutzen verstanden hat. Es ist daher kein Wunder, daß England schon frühzeitig die führende Rolle auf diesem Gebiete übernommen hat, während man in Deutschland die offizielle Medizinalstatistik iahrzehntelang Dilettanten überließ. Selbst heute haben 16 von den 17. deutschen Ländern noch keinen amtlichen medizinischen Statistiker! 